


\section{El temple de ánimo como horizonte de la reflexión: autoexamen, decisión y consideración emotiva}

Ignacio Quepons Ramírez Universidad Michoacana de San Nicolás de Hidalgo

Resumen

El objetivo de este estudio es aclarar la idea de reflexión en el contexto de la explicitación de los horizontes del yo personal y su relación con la noción de "autoexamen", como meditación sobre la propia vida con su valor concreto. Para ello hacemos un balance de la noción de autoexamen o Selbstbesinnung en diferentes escritos de Husserl con la finalidad de aportar una aclaración que permita comprender un sentido amplio de la reflexión, no necesariamente intelectualista, y su lugar en la idea de autoconocimiento como esclarecimiento del sentido de la vida concreta desde una perspectiva fenomenológica.

Palabras clave: Autoexamen, autoconocimiento, temple de ánimo, consideración emotiva.

\section{Abstract}

The aim of this study is to clarify the idea of reflection in context of the explicitly state of the horizons of self and its relationship with the notion of "self-examination", as meditation about the personal life and its concrete value. We perform a balance of the notion of "self-examination" or Selbstbesinnung in several Husserl writings, the aim 
is to suggest a clarification that allows to understand a widest sense of reflection, not necessary in an intellectual register, as well as establish its place in the idea of self-knowledge as clarifying of the sense of the concrete life from a phenomenological perspective.

Keywords: Self-examination, Self-knowledge, Temperature of spirit, Emotive consideration.

$\mathrm{D}$ e acuerdo con la fenomenología de Husserl, la reflexión no sólo explicita la estructura esencial de la subjetividad, sino que descubre, a través de la aclaración de diferentes horizontes de sentido, las determinaciones concretas que constituyen la consistencia de un "yo personal". En el interior de su obra encontramos diferentes sentidos de la idea de reflexión, uno de ellos, utilizado particularmente en sus obras tardías, es la noción de "autoexamen" o Selbstbesinnung, la cual fue convirtiéndose en un proyecto paralelo de aclaración racional del sentido de la vida concreta en relación con la constitución universal del sentido del mundo de la experiencia.

El concepto de Selbstbessinung o "autoexamen" comenzó a utilizarse en la filosofía alemana de finales del siglo XIX y principios del siglo XX, cada vez con más fuerza, como una opción al sentido más intelectualista atribuido habitualmente a la noción kantiana de reflexión. Con Selbstbessinung Dilthey, por ejemplo, se refería a una meditación sobre sí mismo en un registro propio de la consideración vital de la vida como totalidad, en contraposición con la teoría del conocimiento. Husserl, por su parte, utiliza habitualmente una noción amplia de reflexión, la cual es sobre todo la tematización de la vida de conciencia, ya bien en tal o cual carácter 
de su estructura intencional, y toma a la vivencia aislada, o bien con referencia en la vida de conciencia misma en su fluir concreto. ${ }^{1}$

El objetivo de este estudio es aclarar la idea de reflexión en el contexto de la explicitación de los horizontes del yo personal y su relación con la noción de "autoexamen", como meditación sobre la propia vida con su valor concreto.

En la fenomenología es necesario distinguir la referencia a sí mismo en cuanto al carácter indubitable de la vivencia como vivencia. En este sentido entraría la dimensión epistemológica relevante de la reflexión como acceso privilegiado de la conciencia a su propia vida (Hua III/1, 162 ss.). Por otro lado, tenemos un rasgo no incompatible con el primero, pero que permite comprender a la vida como el horizonte abierto y concreto que es.

El concepto husserliano de Selbstbessinung se refiere usualmente a un proceso sistemático y, en esa medida, más amplio (aunque no contradictorio) con el acto representativo de la reflexión. ${ }^{2}$

Cuando Husserl se refiere a un autoexamen fenomenológico (phänomenologische Selbstbesinnung) se trata de algo más que la mera reflexión psicológica como referencia puntual a mi vivencia actual e incluso a la reflexión en sentido fenomenológico, una vez ejecutada la desconexión con la tesis de la actitud natural. Si suspendo la tesis de la actitud natural, puedo referirme a mi conciencia en su pura inmanencia de experiencia, sin asumir los vínculos causales ni la tesis de existencia del mundo real; es decir, puedo referirme a la especificidad de mi vida de conciencia en cuanto tal, pero la vida que analizo es, en cada caso, mi propia vida, incluso una vez ejecutada la reducción. La pregunta que podríamos hacer aquí es: ¿se mantiene mi identidad, es decir, la unidad de mi vida

${ }^{1}$ Ver, por ejemplo, Hua III/1 $\$ \$ 42,45,50,57$; sobre todo hay que tener en cuenta $\$ \$ 77,78,79$.

${ }^{2}$ Algunas apariciones de dicha noción en este sentido se encuentra en sus Meditaciones Cartesianas (Hua I, 116, 179). 
con el acervo de experiencias y el horizonte de referencias axiológicamente relevantes para mí, justo eso que existencialmente hace que mi vida merezca ser vivida? Incluso en actitud trascendental, no hay razones para suspender en primera instancia esa dimensión de la vida fáctica y la correlativa unidad de su "sí mismo" como yo personal. De hecho, el mundo sigue ahí, pero en la reducción trascendental ha sido llevado a su estatus de mero fenómeno de sentido y con ello, en realidad hemos ganado la especificidad del mundo de la experiencia en lo que tiene de propio en cuanto a su confirmación efectiva: ser el horizonte situacional de mi vida. ${ }^{3}$ Así, sin renunciar sin más a la noción de reflexión y sus propios alcances en el interior de la fenomenología de Husserl, quisiéramos dar aquí consistencia a un cierto concepto de autoexamen inspirado en la meditación husserliana sobre la aclaración de sí mismo o lo que él mismo llama "llegar a conocerse" (Hua IV, 249, 252, 270272), el cual, se distingue del sentido de reflexión metodológica dirigido a la explicitación de las estructuras de la conciencia, aunque no es en modo alguno contradictorio con éste.

Para aclarar y dar consistencia a la idea de autoexamen, no sólo como reflexión sobre la vida concreta, sino también como su exploración, como apercepción de su valor existencial, la significatividad valorativa de mi propia existencia como proyecto vital, haremos referencia al papel de los horizontes afectivos de la conciencia de sí que forman, en los cuales se realiza la predelineación de mis motivaciones y mi actuar concreto de acuerdo con las circunstancias de mi

${ }^{3} \mathrm{El}$ resultado del tratamiento de esta cuestión en Ideas I es la idea de un yo puro como polo de unidad al cual se atribuyen todas las vivencias de la corriente de vida. Especialmente en sus Ideas II y más adelante, en sus lecciones de Psicología Fenomenológica de la década de los veinte, Husserl va a requerir la distinción entre el yo puro como puro polo vacío de unidad de las vivencias y su concreción efectiva como yo personal, el cual no es el mero yo puro, sino el yo como resultado de la sedimentación de habitualidades, experiencias en sentido amplio, capacidades y carácter. 
vida. Es así que el objetivo de este estudio es destacar la importancia de la consideración afectiva en el proceso de autoconocimiento y con ello sugerir un cierto sentido práctico de la noción de autoexamen. ${ }^{4}$ La aclaración del sentido de la vida concreta se vuelve entonces la elucidación del sentido de mi vida con relación al horizonte de referencias significativas de mi mundo entorno, manifiestas a través de temples de ánimo y atmósferas del sentimiento. Si bien es verdad, como afirma el propio Husserl, que una tematización explícita en conciencia reflexiva de la vida, como totalidad es imposible (Hua III/1, \$83, 167; Ideas I, 276), también es cierto que a partir de ciertos sentimientos anticipamos el horizonte total de nuestra vida y en ellos vivimos constantemente síntesis intencionales de referencias relativas al mundo concreto y a la plenitud de nuestra vida en este mundo.

En concordancia con las tesis de filósofos contemporáneos, como Charles Taylor, aunque a través de un recorrido de corte husserliano, quisiéramos sugerir una idea del yo como postura moral que en ese sentido relanza el registro intelectualista en el cual se plantea habitualmente la pregunta por autoconocimiento. En efecto, el horizonte o "marco de referencia" situacional, aludido en los debates actuales por filósofos como Charles Taylor, es fundamental en la constitución de la identidad personal y así, el ámbito donde se realiza el esclarecimiento de sentido, presentado en términos de autoconocimiento, es indisoluble de la aclaración de la referencia hacia aquello que nos resulta valioso. La aclaración de los horizontes afectivos de la reflexión, en particular la impronta de los temples de ánimo como la forma concreta en la cual se vive el mundo situacional, forma parte de una cierta reflexión dirigida, no sólo a aclarar las estructuras universales de la subjetividad,

${ }^{4}$ En el sentido de "llegar a conocerse" al que se refiere Husserl, por ejemplo, en Ideas II, cuando distingue la reflexión dirigida al yo puro de la captación de la vida del sujeto en la unidad de un yo personal (Ver Hua IV, \$58, 257). 
sino también a comprender los nexos de motivación y sus contenidos de una subjetividad concreta. ${ }^{5}$ La asunción de los temples de ánimo, como experiencia de sentido, permite descubrir la vida concreta dentro de un horizonte afectivo en el cual se explicita lo más propio e individual de nosotros mismos y dicha experiencia tiene su correlato en el descubrimiento del mundo como horizonte situacional de mi vida. La meditación fenomenológica sobre sí mismo, Selbstbesinnung, puede ser entonces comprendida en términos de la explicitación de los horizontes intencionales de la apercepción de sí mismo y con ello, el proceso de explicitación del sentido concreto de la subjetividad vivido a la par de su desarrollo (Hua IV, 252).

I. Patencia, latencia, nexos de implicación de sentido: aspectos de la intencionalidad de horizonte

El tema fundamental de la fenomenología es la aclaración del sentido de la experiencia, el análisis de su "intencionalidad" (Hua III/1, 74; Hua I, 82). La vida de conciencia es siempre y en cada caso "conciencia de algo", por tal motivo está por entero lanzada a la determinación de una cierta orientación significativa del entorno en diferentes grados y dinámicas específicas. Así, la explicitación de las formas de conciencia, tales como la imaginación, la percepción o el recuerdo, se determina en función de ciertos objetos propios de tales experiencias. El objeto de la percepción es lo percibido en cuanto tal y, en esa medida, los caracteres de ofrecimiento del objeto dado en dicha experiencia son coherentes con cierta mención

${ }^{5}$ Cfr. Ch. Taylor (2006: 70- 85), Aquí se puede ver la semejanza de nuestra posición con el planteamiento de Taylor sobre los "marcos referenciales" de valor como fuentes del sí mismo, dichos marcos referenciales son, en una de sus acepciones, horizontes afectivos en los cuales se explicita la situación vital relativa al yo personal. 
de sentido intencional de la vivencia de percepción. La experiencia de sentido está, a su vez, modulada en diferentes grados de claridad y forma parte de la esencia de la intuición dejar abiertos horizontes de sentido donde se anticipan caracteres o rasgos del objeto todavía por darse o no intuitivos en absoluto, los cuales corresponden, tanto al trasfondo de los sedimentos de nuestra experiencia anterior, como al horizonte situacional en el cual tenemos tal o cual experiencia. Es así que la intencionalidad no sólo se refiere a la mera dirección activa de las vivencias en las cuales se brinda el objeto principal de nuestra atención, sino que en esa misma experiencia hay direcciones de sentido operando de forma latente, constituyendo lo que Husserl llama "el horizonte indeterminado de indeterminación” (Hua I, 82; Walton, 2004). Todo horizonte experiencial es, en primera instancia, un horizonte de patencia y de latencia, es decir, un horizonte de lo dado efectivamente en la situación experiencial concreta en el que se da el objeto de mi interés primario y un horizonte de posibilidades anticipadas relativas, tanto a la experiencia patente, como a nuestro sedimento de experiencias semejantes. La corriente de la temporalidad esencial a la propia experiencia hace que cada objeto de experiencia sea determinado, no sólo respecto de su presencia viva, sino también respecto de todas las experiencias pasadas y una serie de anticipaciones propias de la estructura esencial de la vida de conciencia, las cuales coinciden con su carácter tendente a la trascendencia. A cada percepción de un objeto dado corresponde una anticipación basada en los caracteres que esperamos ver de acuerdo con nuestra experiencia habitual de objetos semejantes o vividos en circunstancias similares. Estas síntesis operativas que intervienen pasivamente en la configuración de los objetos de nuestra experiencia forman horizontes de nexos de sentido. La asociación entre los contenidos sedimentados y los caracteres de la experiencia actual no son necesariamente sucesivos; es decir, la anticipación de sentido basada 
en mi experiencia anterior no proviene de vivencias inmediatas, sino que puede provenir de sedimentos y configuraciones de sentido que forman habitualidades perceptivas, modos de ver, por así decir, en los que estamos habituados a observar y reconocer los objetos de la percepción.

Los horizontes, por otra parte, son fondos a partir de los cuales el objeto perceptivo se destaca gracias a la atención dirigida a él. Para Husserl, la diferencia entre tema y fondo es relativa a la atención. El fondo juega un papel en la determinación de lo destacado, pues el horizonte de fondo no es un mero campo muerto, sino un complejo de referencias implícitas en el que se constituyen las relaciones extrínsecas entre el objeto destacado y los diferentes niveles de contextos, que en última instancia, es el horizonte total del mundo, como "horizonte de horizontes" (Hua I, 97). El fondo perceptivo de cada aparición de un objeto temático está conformado por un horizonte de anticipación de contenidos concordantes, tanto con la experiencia actual, como con las formas en las que estamos habituados a vivir el objeto destacado.

Los horizontes también son sistemas de asunciones presupuestas bajo la modalidad "yo puedo" (Ich kann) relativas a capacidades prácticas adquiridas por un yo concreto, el cual está vinculado con la posibilidad de anticipar la consecución de una vivencia perceptiva. Los vínculos inferenciales de los entrelazamientos de la experiencia son vínculos de motivación. Todo el campo perceptivo se forma sobre la base de un complejo de potencialidades de movimiento de mi cuerpo, en el cual se anticipa el mundo dado como horizonte de resolución práctica de acuerdo con mis capacidades adquiridas (Hua I, 109; Hua XI, 119; Hua IV, 223). De igual forma, la captación de un objeto del mundo circundante o una situación concreta pone en juego el sistema de motivaciones por las cuales se capta la significatividad del objeto, en relación con otros sentidos, como comprender la puerta como acceso, captar 
el picaporte como algo dispuesto a ser tomado por la mano y que sirve para abrir la puerta, etc. ${ }^{6}$

Las síntesis de habitualidad no son exclusivas de las formas perceptivas y en general de las vivencias cognoscitivas, hay también formas de anticipación, remisión y nexos de sentido en la esfera de la acción, la volición y el sentimiento. También los objetos de nuestras preferencias valorativas y las motivaciones de nuestra acción libre, sus metas, están prefiguradas gracias a horizontes que se refieren a determinaciones de sentido emotivo. El éxito o fracaso en nuestras posibilidades relativas a la acción, las experiencias placenteras o dolorosas, las alegrías y las tristezas, van configurando también horizontes indeterminados de determinación posible de experiencias semejantes a lo largo de nuestra vida concreta.

II. Intencionalidad del temple de ánimo: fondo emotivo y nexos de implicación pasiva de sentido en la esfera del sentimiento. La anticipación afectiva de la vida como totalidad concreta

La fenomenología de la vida emotiva no es la caracterización psicológica de los sentimientos sino el estudio de su carácter de unidad de sentido en la experiencia, es decir, el análisis de las relaciones intencionales específicas que anidan en ellos. Es gracias a nuestra dimensión emotiva que podemos captar el mundo como un mundo de situaciones con valor En ese sentido, el análisis fenomenológico está dirigido al estudio de la esfera axiológica como unidad de rendimientos intencionales. El mundo del valor es un mundo que viene al caso en un registro diferente al de la mera percepción; desde el punto de vista de la actitud valorativa, el mundo es percibido con un "sentido espiritual", es decir, resulta axiológicamente

${ }^{6}$ Véase por ejemplo: Hua IV, 238. 
relevante; nos interesa, nos incumbe, nos importa y la motivación de este interés es un movimiento del ánimo.

La estructura de horizonte de la intencionalidad relativa a los temples de ánimo no sólo es una peculiar forma de apercepción del mundo entorno, sino también el campo de unificación del tejido emotivo de la vida concreta. A partir de síntesis de habitualidades, se forman sistemas de anticipación emotiva y resolución práctica, y se configura la significatividad de la propia vida. En ese sentido, la meditación sobre sí mismo no es el mero acto reflexivo que pone a la vida como un objeto acabado ante sí, sino el proceso de indagación que explicita los sistemas de implicación emotiva siguiendo las pautas de la intencionalidad de horizonte contenidas en los temples anímicos.

A lo largo de la obra de Husserl es posible encontrar diferentes descripciones del temple de ánimo como fondo o atmósfera emotiva de la situación concreta (Hua XXXVII, 327; Hua IX, 415; Hua XXXVII, 327; Hua XIX/1, 409-410; Hua XXXVII, 342-346; Ms. A VI 8 I/50a "H” 26; Ms. A VI 8 I/66a “36"; Ms. A VI 12 I/268a “42”; Ms. A VI 8-I, A VI 12, A VI 34, entre otros). Pero también, particularmente en sus manuscritos a partir de la década de los años treinta, tenemos un énfasis en la idea de temple de ánimo como un rasgo esencial en la unidad de la vida, no sólo relativa a la apercepción del mundo.

Uno de los aspectos relativos al temple de ánimo es la anticipación emotiva (Gefühls-Antizipation), la cual es una capacidad del sujeto concreto en relación con el sentimiento que le despierta la situación vivida actual. Este vivir puede ser un sentir a la manera de un presentir, en un sentido muy literal, pues se trata de una anticipación, una expectativa en el sentimiento motivada por la vivencia actual. La mención de valor vacía, a la que se refiere aquí, es precisamente un horizonte aperceptivo que anticipa la plenitud del valor. La apercepción del valor es siempre una apercepción emotiva, de 
modo que podemos llamar aquí a esta forma de referencia, sin mayor justificación: horizonte afectivo (Ms. A VI 34 (1931) 24 b).

Un rasgo de las descripciones sobre los temples de ánimo, particularmente entre 1931 y 1933 , es el énfasis en su carácter temporal $y$, con ello, cierto horizonte de anticipación emotiva de los temples en el cual se compromete el valor de la vida concreta. En particular, destaca la descripción del temple del "cuidado" o "preocupación”, en alemán: Sorge. El cuidado es una forma de anticipación de horizonte dirigida a la vida como totalidad y está vinculada a experiencias de expectativa emotiva propias de vivencias, como el miedo o la esperanza. A diferencia de otras expectativas o anticipaciones emotivas relativas a tal o cual aspecto o carácter de la valiosidad, el "cuidado" anticipa lo que puede llegar a pasarnos en cuanto sujetos concretos y a través de esa anticipación se mienta también, aunque de forma implícita, el valor de la propia vida como totalidad y su propio horizonte situacional. En el manuscrito E III 6 de 1933, Husserl continúa la exploración del tema de la preocupación o "cuidado", esta vez explícitamente con relación al problema de la existencia (Existenz). ${ }^{7}$ En su ejemplo, Husserl se refiere a un comerciante en peligro de quedar en bancarrota. ${ }^{8} \mathrm{El}$ comerciante, en cuanto tal, asume la eventualidad del fracaso de la empresa que define su profesión como algo en lo que está en juego su propia "existencia". Este comerciante, dice Husserl, tiene un horizonte abierto de posibilidades para encontrar una nueva profesión; es decir, se puede asumir a sí mismo de otra manera, puede tener una actitud diferente hacia el porvenir; no obstante, la even-

${ }^{7}$ Husserl estudió la obra temprana de Heidegger, especialmente en el período que va de 1926 a 1933. La constancia de su estudio está en todas las notas de los márgenes que encontramos en sus ejemplares personales de Ser y Tiempo y Kant y el problema de la metafísica. A pesar de la evidente proximidad entre la descripción de fenómenos semejantes entre ambos, no hay testimonios explícitos de Husserl en referencia al particular en la obra de Heidegger.

${ }^{8}$ Este ejemplo aparece también en el citado Ms. A VI 34, de 1931. 
tual situación de quiebra de su empresa lo coloca en la situación de hacerse cargo del sentido de su proyecto vital como totalidad; es así que vive su vida con esperanza vital (Lebenshoffnung), con cierta expectativa emotiva sobre los acontecimientos venideros de su vida y se encuentra de momento en la preocupación o cuidado vital (Lebenssorge), le preocupa hacia dónde puede orientar su vida, qué será de él. El cuidado, para Husserl, es un modo del sentimiento semejante a la modalización de la creencia en la esfera dóxica, el cual juzga de acuerdo con lo que se anticipa como probable y emotivamente relevante, como horizonte de posible fracaso. Lo importante aquí es que, a diferencia de la mera creencia modalizada de probabilidad o certeza, la dimensión del interés sobre lo que se tiene como cierto o probable es relevante para la ejecución de una acción en consecuencia. ${ }^{9}$ El cuidado (Sorge) es el modo de sentimiento (Gefühlsmodus), por el cual surge la modalización de la actividad y de la cual el pre-señalamiento existente de horizontes de posibles malogros, dentro de los cuales se desarrollan las líneas de previsiones y ciertos éxitos, de los éxitos bajo corrección. ${ }^{10}$

En cuanto sujeto concreto, siempre intento hacer lo mejor que puedo con mi situación vital, anticipo el fracaso, vislumbro el horizonte posible de mi éxito. Muchas veces me preocupo, como resulta evidente, sobre aquello que no depende de mí. Si mi equipo de futbol desciende, me preocupa la situación de mi país, el futuro de la economía mundial; dicho de otro modo: anticipo

${ }^{9}$ Se trata de una instancia de diferentes niveles de anticipación emotiva sobre el horizonte del eventual fracaso en mis aspiraciones prácticas. Sobre el particular, ver Roberto Walton (2004: 238).

${ }^{10}$ Ms. E III 6, 3a, "Die Sorge ist der Gefühlsmodus, der aus der Modalisierung der Aktivität entspringt und aus der bestätigen Vorzeichung des Horizontes möglichen Missligens, innerhalb dessen die Linien des doch voraussichtlichen und gewissen Gelingens, des Gelingenes unter Korrektur, verlaufen". Agradecemos al Dr. Ulrich Melle, Director de los Archivos Husserl, el permiso para citar manuscritos inéditos. 
posibilidades que me incumben o me importan. Naturalmente, la posibilidad fundamental que anula todo el horizonte de mis aspiraciones es la de mi muerte. ${ }^{11}$

En Junio de 1933, Husserl medita sobre el tema del miedo a la muerte (Todesangst). En esta reflexión complementa lo dicho dos años antes sobre el tema de la preocupación sobre la vida. La vida es un permanente cálculo entre el eventual fracaso y el accidente, bajo los cuales, se mantiene la tendencia hacia la auto-preservación.

En la vida cuenta que se presentan accidentes molestos en el detalle, aniquiladores del proyecto del caso, condicionantes de fracasos, sobre el suelo de una general certeza de autopreservación (Selbsterhaltungsgewissheit). Así, a la afirmación de la vida pertenece un descontento en el detalle. Este obstaculiza en verdad el sentimiento general, el temple de satisfacción; uno se pone de mal humor, pero está sin embargo seguro de finalmente reponerse del obstáculo, de vencerlo. El mal humor presupone pues una certeza vital unitaria, certeza de "poder vivir", y emocionalmente $<$ el> 'temple' del humor positivo. 'Éste' implica la certeza de horizonte de la supresión del descontento mediante el temple en otro sentido positivo, agradable, placentero. Pero ambos presuponen como momento singular, como algo momentáneo en el horizonte temporal del temple, precisamente el temple total como algo en totalidad positivum, la totalidad del temple, que como unidad del temple tiene el modo de la certeza. Aquí hay sobre este basamento o más bien en esta forma universal del temple, diferentes formas particulares y formas de transición que describir. ${ }^{12}$

\footnotetext{
${ }^{11}$ Esta dimensión, como es bien sabido, es explorada de forma magistral, con consecuencias que no es el caso explorar en este ensayo, por Martin Heidegger, en su obra monumental Ser y Tiempo.

12 "Zum Leben rechnet, dass im Einzelnen störende, das jeweilige Vorhaben zunichte machende Zufälle, Misslingen bedingende, auf dem Boden einer allgemeinen Selbsterhaltungsgewissheit eintreten. Also zur Lebensbejahung gehört im Einzelnen Unzufriedenheit. Sie hemmt zwar das allgemeine Gefühl, die Stimmung der Zufrie-
} 
El cálculo vital respecto del horizonte anticipado de eventuales fracasos y accidentes se hace siempre desde la certeza general de la preservación de sí. El temple de la preocupación o cuidado indica la preservación de la vida en su consecución, a la manera de un horizonte de implicación de sentido. A pesar de la insatisfacción que inhibe el sentimiento general o temple de satisfacción, hay una cierta seguridad tácita, cierta creencia implícita en que las cosas pueden ser de otra forma. Todo ello supone un temple de ánimo positivo, podríamos decir: la confianza en la superación de los obstáculos a partir de un sentido positivo.

Existe un temple de ánimo como horizonte en el contexto de la asunción de la propia vida, la existencia, como una vida en peligro de ser afectada por el infortunio, la desgracia, el accidente y, en última instancia, la muerte. Resulta interesante que para Husserl el temple de ánimo, como horizonte de fondo, es en este caso una tendencia a la perseverancia, a la superación de los obstáculos, desde la cual se anticipa, emotivamente, un horizonte de eventual fracaso de nuestra empresa y respecto del cual se extiende la anticipación de mi vida total en la forma de una preocupación. Me preocupa lo que puede pasar conmigo.

El tema de la muerte es una de las experiencias límite cuyo sentido, en fenomenología, no es fácil de explicitar. Como señala Ja-

denheit, man wird verstimmt, aber ist doch gewiss, schließlich über die Hemmung hinauszukommen, sie zu überwinden. Verstimmung setzt also eine einheitliche Lebensgewissheit, Gewissheit «Leben zu können», und gefühlsmäßig <die> «Stimmung" positiver Gestimmtheit voraus diese impliziert die Horizontgewissheit der Aufhebung der Missstimmung durch die in einem anderen Sinn positive, wohlige, lustige Stimmung. Aber beides setzt als einzelnes Moment, als Augenblickliches im Stimmungszeithorizont, eben Gesamtstimmung als ein in Totalität Positivum voraus, die Stimmungstotalität, die als Stimmungseinheit den Modus der Gewissheit hat. Hier gibt es auf diesem Untergrund oder vielmehr in dieser universalen Stimmungsform verschieden zu beschreibende Sonderformen und Übergangsformen" (Ms. E III 6/5a). 
mes Dodd, la muerte, el sueño y el nacimiento son para Husserl los límites en los cuales se hace presente al yo su propia vida, como un campo de existencia personal (Dodd, 2010: 62). Por nuestra parte, hemos señalado algunos manuscritos donde aparece la referencia intencional de la preocupación, como una disposición manifiesta en un temple que me entrega mi vida, como vida personal, desde la perspectiva de su totalidad concreta: la mónada que en cada caso soy. La forma de anticipación de la preocupación o cuidado no es una anticipación reflexiva, al menos no lo es en principio, sino que opera a manera de un horizonte no temático de carácter afectivo.

\section{Autoconocimiento como esclarecimiento de la situación vital: horizontes de la reflexión $y$ el autoexamen}

Una vez aclarado el carácter de horizonte propio de los temples de ánimo, en particular el temple del cuidado, en el cual se anticipa y se mienta tácitamente el valor de la vida como totalidad, volvamos al tema de la meditación sobre sí mismo o autoexamen, la Selbstbesinnung. La meditación sobre sí mismo es para Husserl un proceso reflexivo continuado de exploración de sí, se trata justo de un "auto-examen", el cual no tiene necesariamente un sentido moral, sino que puede tratarse de la exploración de los diferentes aspectos y características de la vida concreta en general. No obstante, y en esto es muy importante la distancia entre Husserl y la filosofía kantiana, para Husserl la subjetividad trascendental no es una pura forma, sino justo la vida fáctica como mónada concreta, pero asumida en su especificidad experiencial. El mundo, este mismo mundo tiene sentido y valor para mí. La explicitación de este sentido en el cual se constituye la esencia misma de lo que es el mundo, tal y como se manifiesta en la experiencia, es una explicitación de su sentido en mi propia experiencia. La formación del 
sentido de mi vida fáctica involucra naturalmente también mi propia historicidad y los sedimentos de experiencia resultante de mi interacción con otros sujetos fácticos que co-constituyen el horizonte de mundo de su experiencia común conmigo. Es así que, visto desde el punto de vista de la vida trascendental fáctica que somos, la Selbstbesinnung es una meditación sobre mi propia vida y la formación de su identidad, la cual incluye, en síntesis de implicación, las menciones correspondientes a las unidades de sentido intersubjetivo e histórico. Eso que soy, como yo personal fáctico, lo soy en relación al resultado de experiencias de interacción con los demás y la herencia de nuestra historia común; en cada caso se trata siempre de experiencias mías y es el contenido de esa herencia algo que vivo como mío y reconozco como propio, de otra forma no serían constatables, sino que, eventualmente, también serían irrelevantes.

Entonces, la idea de "autoconocimiento" no es la mera representación de las formas generales del sujeto cognoscente a la manera de la filosofía de Kant, sino también el recorrido de explicitación de sentido, para Husserl, racional, a través del cual confirmamos la racionalidad de la experiencia que somos nosotros mismos. Experiencia, una vez más, relativa a la dimensión intersubjetiva e histórica de nuestra condición como subjetividades concretas.

En este sentido, a pesar de las profundas diferencias entre Husserl y Dilthey, podemos encontrar afinidades entre el concepto que intentamos trazar aquí desde Husserl, con la noción más explícita de Dilthey de Selbstbesinnung, ${ }^{13}$ como meditación sobre la vida concreta como totalidad y no sólo como instancia intelectual de representación. Existen suficientes motivos para delinear un sentido especial y particularmente fenomenológico de un tipo de reflexión como Selbstbesinnung, orientada no a la aclaración de los aspectos generalizables de la estructura de la subjetividad, sino a la

${ }^{13}$ Véase Renato Cristin (2000: 45- 65) y Alexis Gross (2011: 7- 15). 
significatividad de nuestra propia vida, la cual, además funciona a partir de las pautas de análisis genético de horizontes propuestas por el propio Husserl.

La reflexión comprendida como proceso de explicitación o auto-examen, en el sentido de Selbstbesinnung, en lugar de plantear la figura del sí mismo a partir de una representación, hace explícitos los niveles de formación de horizontes implicados en la constitución de la identidad del sujeto. Dichos niveles involucran contenidos de orden axiológico y de la esfera de la acción, así como los sedimentos resultantes de la interacción intersubjetiva y la historicidad de la subjetividad fáctica.

Ya en su tratado inédito, Ideas II, Husserl hacía algunas precisiones relativas a la diferencia entre la conciencia, como pura corriente de vida con su yo puro, y el yo personal, como una unidad constituida a través de un proceso de desarrollo. La autoconstitución de la subjetividad como mónada concreta implica, en el despliegue de su desarrollo constituido por tomas de posición, habitualidades y capacidades, las cuales pueden ser reconocidas en evidencia como justo todo eso que "yo soy", con mis potencialidades, mis aspiraciones y proyectos (Hua IV, 248). En este contexto Husserl señala que antes de ejecutar la reflexión encontramos justo a un yo personal irreflejado, con sus predaciones y referencias al mundo circundante, asumido como su mundo circundante, con sus bienes, valores y referencias significativas. Este yo puede asumir en la reflexión o percepción de sí mismo un modo particular del yo vivo en el nexo general de su vida. Yo percibo que he vivido tal o cual circunstancia, que he visto cierto objeto antes, que también puedo recordarlo. Esta dimensión se extiende a toda la esfera de la vida de conciencia, también a la vida afectiva, "una alegría que me movió, aún me mueve" (Hua IV, 248).

En estas páginas, a diferencia del tratamiento de la reflexión (comprendida como método de investigación dirigida al análisis 
de lo apodíctico en las vivencias), Husserl se refiere a la reflexión del yo personal y a la percepción de las particularidades y comportamientos concretos y, en esa medida, fácticos del despliegue de los horizontes de un yo concreto en su vida. ${ }^{14}$

En tanto, cuando me muevo así en actos de reflexionar vivos, irreflejados, en campos de reflexión (de las subjetividades objetivadas), EXPERIMENTO con ello cómo me "comporto" en diferentes circunstancias subjetivas, esto es, con referencia a mi esfera de las predaciones respectivas (de mi mundo circundante en un sentido amplísimo); y si me adentro en el entrelazamiento de las motivaciones de mi cogito, en las intencionalidades abiertas y ocultas de la motivación, entonces experimento cómo soy motivado por ellas y cómo SUELO estar motivado por ellas, qué ÍNDOLE PECULIAR EXPERIMENTABLE poseo EN GENERAL en cuanto sujeto de motivación de estas circunstancias motivantes (Hua IV, 249).

El análisis de mi comportamiento en diferentes circunstancias subjetivas conlleva a la consideración de los nexos de motivación, cuya estructura es la de una intencionalidad de horizonte, como sistemas de anticipación sobre la base de sedimentaciones de experiencias semejantes. La evaluación de mi comportamiento en "diferentes circunstancias subjetivas" supone el sedimento y las síntesis de asociación entre los aspectos concordantes o semejantes de las situaciones, así como el contexto situacional, en sentido amplio, el mundo circundante concreto, el cual es también un horizonte de

${ }^{14}$ Esto no quiere decir que dicho análisis no haga explícitas estructuras trascendentales, todo lo contrario, las formas de configuración del sentido concreto de la subjetividad son formas trascendentales comunes a toda vida de conciencia, en general instanciada y únicamente dada con evidencia en cada subjetividad fáctica, las cuales, no obstante, pueden ser expresadas en enunciados de esencia, de acuerdo con la doctrina fenomenológica. 
sentido co-asumido en el ofrecimiento de mi vida en situación al movimiento de la reflexión que la analiza.

Un poco más adelante, en el mismo texto distingue explícitamente entre los dos sentidos de reflexión: el primero, en sentido teórico, que apunta al yo puro; el segundo, denominado como "experiencia temática reflexiva", base del movimiento que aquí proponemos como "autoexamen", que aparece como una reflexión basada en la apercepción de la experiencia desarrollada sobre la cual se conforma la unidad del yo personal.

Distinguimos, pues, la REFLEXIÓN DEL YO PURA, de la reflexión sobre el yo puro que pertenece por esencia a todo cogito, la EXPERIENCIA TEMÁTICA REFLEXIVA, sobre la base de la apercepción de la experiencia desarrollada, cuyo objeto intencional es este yo empírico, el yo de la intencionalidad empírica, como experiencia de sí mismo, del YO PERSONAL, con referencia en los nexos de experiencia en los cuales este yo personal (o sea, con referencia en los actos que ejecuta en las circunstancias motivantes pertinentes) se acredita según sus "PECULIARIDADES PERSONALES” O RASGOS DE CÁRACTER (Hua IV, 249).

La idea del "autoconocimiento" no se realiza en un acto aislado de reflexión, ni se cumple en la confirmación evidente del yo puro en la unidad de su corriente de vida. Como menciona Husserl, más adelante, en Ideas II, el conocimiento de sí, como subjetividad concreta, se realiza a la par del desarrollo vital del sujeto que reflexiona sobre su vida en situación. "Alguien no se 'conoce', no 'sabe' lo que es, LLEGA a conocerse. La experiencia de sí mismo, la apercepción de sí mismo, se amplía constantemente. El 'llegar a conocerse' es uno con el desarrollo de la apercepción de sí mismo, de la constitución del 'sí mismo', y ésta se ejecuta a una con el desarrollo del sujeto” (Hua IV, 252). 
En los manuscritos tardíos de los años treinta encontramos, en varias ocasiones, la referencia a la noción de Selbstbesinnung, en un sentido más o menos unitario. ${ }^{15}$ En casi todos los casos encontramos justo la idea de un autoexamen o meditación sobre sí mismo, es decir, un proceso radical de esclarecimiento del sentido de la subjetividad asociada a experiencias como autoconciencia, visión de sí mismo (Selbstanschauung), percepción de sí mismo (Selbstwarhnemung) en las cuales se realiza un conocimiento de sí (Selbsterkenntnis) cuyo objetivo final es, en última instancia, la respuesta a la pregunta "quién soy yo esencialmente"; dicha pregunta remite, en el proceso reductivo que efectúa el autoexamen, al presente vivo que en cada caso soy yo mismo (Ms. C 7 Texto 36; Hua Mat. 8, p. 139).

Nuestra propuesta aquí es que el proceso de la automeditación o autoexamen puede efectuarse a partir de un esclarecimiento del sentido implicado en los horizontes intencionales. De tal manera, dado el carácter de horizonte de los temples de ánimo, también es posible plantear la posibilidad de un esclarecimiento de sí, como vida concreta, a partir de la explicitación de horizontes emotivos en los cuales se constituye la situación vital respecto de la cual mi propia identidad, como persona, y el valor de mi vida adquieren sentido. En sus manuscritos tardíos podemos encontrar al menos una ocasión en la que el propio Husserl vincula el análisis de horizontes con la actividad misma de la meditación sobre sí mismo.

En el texto 7 del manuscrito C 6, Husserl señala que la meditación sobre sí mismo es una actividad del yo en relación a sí mismo, la cual, como toda actividad, tiene un pre-tener (Vorhabe); es decir, un proyecto o plan, algo que asume anticipadamente. En ese sentido, dice Husserl, nunca tengo con ello algo frente a mí, sino eventualmente a mí mismo y quizá no sólo ocasionalmente. El ma-

${ }^{15}$ Sobre Selbstbesinnung ver: Ms C 4 Texto 21, Hua Mat 8, p. 88, así como Meditaciones Cartesianas, (Hua I, 116, 179). 
nuscrito trata en la primera parte sobre la dimensión del proyecto, desde el punto de vista de la volición, pero en cierta parte vuelve sobre la cuestión del autoexamen y señala los diferentes sentidos de la idea de "meditación" (Besinnung):

La meditación (Besinnung) es polisémica: 1) el percatarse-de-unproyecto-actual (Einer-Vorhabe-aktuell-Innewerden) que ya tiene; vuelve a mí en el sentido de que lo recuerdo: se vuelve de nueva cuenta actual. 2) "Medito" (Ich besinne mich) en el sentido más habitual y amplio, despierto, "traigo a consideración” el día, el horizonte de mi proyecto, eventualmente, sobre todo, se vuelve actual mi "horizonte vital" (Lebenshorizont). La meditación sobre el excelente proyecto del día, mi vida y así particularmente en relación a cualquier proyecto especial, ya en actualidad, en cuanto a su qué pero la meditación se vuelve actual en la forma de que me vuelvo consciente de lo dado de antemano; pero con ello estoy dirigido al qué (Ms. C 16, Texto 67 Hua Mat 8, 303).

Finalmente, tenemos la reflexión en torno al autoexamen mismo como meditación relativa al mundo de la experiencia en sus horizontes. En este fragmento se sugiere precisamente la posibilidad de analizar la meditación sobre sí mismo como un proceso donde interviene la intencionalidad de horizonte y, en ese sentido, como una explicitación del mundo de experiencia:

Meditación sobre sí mismo (Selbstbesinnung), a saber, meditación con respecto al mundo como es puramente válido desde mi experiencia, a mí en cada caso, enuncia aquí: descubrimiento sistemático del horizonte, descubrimiento sistemático del sentido del ser del mundo, de hecho como válido en cada caso desde mi experiencia, el cual ha nacido como co-mención en la horizonticidad (Horizonthaftighkeit). Ejecuto aquí, como resulta obvio, la interrogación de la mención de horizonte, en cuanto a lo más general, así ella apunta a lo general-formal de este mundo, sobre todo, en 
cada caso, como mundo de experiencia, tal como yo, por consiguiente, lo encuentro ahora, como siempre lo he encontrado y como lo encontraré, mejor dicho, como tengo que encontrarlo. ${ }^{16}$

La meditación sobre sí mismo se despliega, según hemos sugerido por nuestra parte, de acuerdo con diferentes horizontes en los que se explicita el sí mismo como núcleo de la unidad e identidad de mi vida concreta; a dicha unidad dinámica cabe llamar "yo personal", la cual se descubre en explicitación de horizontes: conocerse es ir interrogando los horizontes de la experiencia de nosotros mismos. La experiencia que somos es siempre experiencia relativa al mundo, por tal motivo, la meditación sobre sí mismo es a su vez siempre, y en cada caso, meditación sobre el sentido del mundo concreto de mi experiencia. Si llevamos la cuestión al nivel del temple de ánimo, el mundo se manifiesta como un mundo de la vida originariamente vivido, como mundo con valor y horizonte abierto de mi situación vital. La meditación dirigida a la explicitación del temple de ánimo, como experiencia de sentido, como horizonte afectivo, descubre al mundo como el fondo situacional de mi vida concreta. De modo que meditar sobre sí mismo es a su vez explicitar el sentido de toda la situación histórica en la que vivo y con ello hay una dirección relativa a la comunidad intersubjetiva y al momento histórico que vivo.

${ }^{16}$ Ms. C 7 Texto 37 Hua. Mat. 8, p.141. "Selbstbesinnung, nämlich Besinnung hinsichtlich der Welt als der mir jeweils rein aus Erfahrung geltenden besagt hier: systematische Enthüllung der Horizonte, Enthüllung des Seinssinnes Welt eben als der jeweils aus Erfahrung geltenden, der in der Horizonthaftigkeit als Mitmeinung geborgen ist. Vollziehe ich, wie hier selbstverständlich, die Befragung der Horizontmeinung in Hinsicht auf das Allgemeinste, so zielt sie auf das Formal-Allgemeine dieser Welt als jeweilige Erfahrungswelt für mich überhaupt, als wie ich sie also jetzt finde und immer wieder gefunden habe und finden werde bzw. muss finden können" 


\section{Autoexamen y análisis de implicación de horizontes} de los temples de ánimo: aspectos de una fenomenología de la vida concreta.

La noción de Selbstbesinnung permite recuperar un matiz no incompatible con la reflexión, pero que integra una dimensión más amplia respecto del registro epistemológico habitual de los actos reflexivos; no sólo puedo volverme reflexivamente hacia mi vida, sino que en ese volverme puedo efectuar un "autoexamen", realizo en él una consideración, una ponderación valorativa sobre mí mismo y el valor de mi vida, con lo cual se le da un cariz diferente a la figura del autoconocimiento, se hace de él un autoexamen en el cual es relevante el marco referencial axiológico correspondiente a mi vida concreta y en el cual se constituye la unidad de mi yo personal.

¿Cómo procede la meditación sobre sí mismo en estos términos? La pauta fenomenológica fundamental, destacada por Husserl en Meditaciones Cartesianas, es que la vida, si bien en cada caso apodíctica e indudable, también es siempre un horizonte abierto que no se ofrece de un solo golpe de la mirada, sino que se descubre a partir de un proceso de explicitación de sus horizontes.

Hay una relativa inadecuación de mí para mí mismo con relación en el acervo de experiencias que constituyen mi propio pasado personal, así como el sentido de mis propias expectativas y aspiraciones vitales. Este horizonte latente no se encuentra, desde un punto de vista fenomenológico, "oculto", sino inatendido y se manifiesta constantemente en lo que Husserl llama una coloración emotiva del entorno, un temple de ánimo. La Selbstbesinnung no sólo es una reflexión analítica de las estructuras de la conciencia, sino también un proceso meditativo que explicita las implicaciones de un sentido intencional tácito, cuya exhibición sensible es la atmósfera emotiva de la situación, el "cuasi-correlato" objeti- 
vo de cómo me "encuentro" (sich befinden); aunque el objetivo es otro, no obstante, se procede de acuerdo con las mismas pautas del análisis intencional, que son tema de la fenomenología en sentido estricto.

El autoexamen que toma como hilo conductor los horizontes de implicación de sentido de los temples de ánimo podría explicitarse en el análisis de la formación del carácter y la resolución en una alternativa de vida como afirmación de la voluntad.

En Ideas II, Husserl distingue entre un sujeto empírico de actos y su noción racional de persona. Se trata de un sujeto ponderado desde el punto de vista, no sólo psicológico, sino de la razón. En esa medida es el sujeto que es "responsable de sí mismo". No se vive, como en reiteradas ocasiones señala Husserl, como una mera corriente de vida, sino como una corriente que asume sus vivencias como vivencias de un yo (Hua IV, 265). Además, puede asumirse a sí mismo como sujeto de razón, es decir, libre, y determinarse a sí mismo en la figura de la autoresponsabilidad. Ello supone el sentido de autoexamen como figura de conocimiento de sí, al cual nos referimos aquí. ${ }^{17}$

El sujeto, en cuanto mónada concreta, es el resultado de su experiencia y como tal tiene una apercepción de sí mismo como un yo: una autoconciencia empírica. En esa autoconciencia se asume como la unidad constituida por sus "tomas de posición", tanto de manera activa, así como el resultado sedimentado de tomas de posición pasadas y sus costumbres y sus capacidades adquiridas. También se apercibe a sí mismo como cierto de sí en determinado

${ }^{17}$ La idea de conciencia de sí (Selbstbewusstsein) y autodeterminación de la voluntad orientada racionalmente es un tema clásico de la filosofía alemana, después de Kant. Es posible apreciar esta idea, tanto en la filosofía de Fichte, que fue objeto de estudio del último Husserl, como de Hegel. El movimiento de la autoconciencia no es sólo conocimiento de lo que se es, sino también adquisición de "conciencia" en sentido moral y, con ello, consumación de la libertad. Esta idea además hunde sus raíces en la filosofía de Spinoza. 
carácter, al punto de que puedo variar en la fantasía diferentes situaciones y pensar cómo me comportaría en ellas. Mis eventuales reacciones o tomas de posición, dice Husserl, no son meras inferencias de expectativa, sino que surgen de caracteres intencionales que comparo con la apercepción de una cosa y el sistema de experiencia en el cual se despliega su aparición, es decir, en la unidad de su horizonte de expectativas "posibles" de acuerdo con nuestra experiencia de la cosa que se trate. "En todo caso, todo rasgo del objeto intencional remite a experiencias similares anteriores; no hay en la aprehensión de la cosa nada por principio nuevo. Si este fuera el caso, entonces sería ya el comienzo de la constitución de un nuevo estrato unitario" (Hua IV, 266).

Ahora bien, como señala un poco más adelante, tampoco soy una mera cosa que en circunstancias iguales reaccione igual, sino que en ciertas circunstancias fui motivado a actuar de cierta forma y en el tránsito entre dicha circunstancia y otra, soy otro. La motivación puede ser incluso la misma, pero la fuerza de la motivación puede variar. Husserl pone el ejemplo de los arrebatos propios de la juventud y la contención, producto de la experiencia de las motivaciones del viejo, que conoce las consecuencias. Así, se pregunta Husserl: ¡cómo llego a conocer los soportes de la motivación, sus direcciones, sus fuerzas si mi vida cambia? Su aclaración apunta a una explicitación del sentido de la voluntad libre, justo como institución del sujeto responsable y, en ese sentido, cierto de sí. La voluntad comprendida como auténtico querer se orienta desde el yo, como resolución o decisión, no me dejo llevar por el mero impulso. La voluntad más auténtica, orientada a fines racionales, se ejecuta como tal gracias a la ponderación de la consecuencia entre dichos fines y la experiencia de valores elevados. "El valor más elevado lo representa la persona que habitualmente le confiere 
la mayor fuerza de motivación a la resolución genuina, verdadera, válida, libre" (Hua IV, 268). ${ }^{18}$

No obstante, la formación de dicha habitualidad supone justo una meditación, sobre las motivaciones y sus nexos, basada en la "experiencia". Como dice Husserl más adelante, en el mismo parágrafo de Ideas II, "La persona se forma por «experiencia»" (Hua IV, 271). Además, volviendo a nuestros análisis anteriores, dicha ponderación de la vida se realiza en el horizonte de vivencias afectivas que descubren, en anticipación afectiva, mi vida como totalidad; por tanto, forman parte también de los nexos motivacionales que me llevan a actuar en consecuencia con mi situación actual; de hecho, el hábito de volverse sobre la vida en un autoexamen requiere también el temple, en el sentido de hacerse a un hábito de la disposición afectiva, del carácter. El cuidado (Sorge) se me presenta como una anticipación emotiva de mi vida como totalidad que despliega de forma no temática su valor. La ponderación de mi situación vital y consecuente decisión, en el sentido no de elegir tal o cual decisión, sino de determinarse a sí mismo bajo la forma de la autoresponsabilidad radical, supone el hábito de la formación

${ }^{18}$ Husserl explora una fenomenología de la voluntad, enteramente consecuente con su exposición en Ideas II, en un excurso a su lección de Ética de 1908/ 09, 1911, intitulado precisamente: "Fenomenología de la voluntad" (Hua XXVIII, S. 102-125). Dichas lecciones corrieron a la par de los preparativos, tanto de Ideas I como de la redacción de diferentes manuscritos de investigación sobre la misma temática, particularmente con relación en su lectura de Alexander Pfänder. Ver la introducción de Karl Schuhmann a su edición de Ideas I (Hua III/ 1, XVIII- XX), donde hace alusión a diferentes manuscritos integrados más adelante, poco después de la última revisión de Ideas II, en el proyecto de publicación, igualmente inconcluso, conocido como Estudios sobre la estructura de la conciencia. El texto mecanográfico de Landgrebe (Ms. M III, 3 I-III) de dicho proyecto fue realizado tres años después del de Ideas II, de 1924, pero incluye manuscritos de ese año. La tercera parte de sus Studien constituye la fuente más amplia de investigaciones de Husserl sobre la fenomenología de la voluntad enteramente coherente con lo manifiesto en Ideas II. 
del carácter, la meditación sobre sí mismo, la exploración de los horizontes de mis motivaciones afectivas y el fortalecimiento, en consecuencia, de mi voluntad.

El aporte del modelo de explicitación de horizontes consiste en destacar la vida afectiva en términos de la implicación de otras conciencias en toda la conciencia; es decir, toda vivencia intencional no sólo está dirigida a un objeto, sino que además está vinculada con otras vivencias. Todo sentido llama a otro sentido semejante o implica un sedimento de sentido habitual, el cual determina la forma de lo dado actualmente. La corriente de vida, desde el punto de vista de la actitud emotiva, siempre es una mezcla de diferentes sentimientos; sólo a través del análisis se puede obtener la forma aislada de cierta regularidad de la vida a la cual es posible adjudicar el nombre de mera alegría o tristeza. Los temples de ánimo son el fondo pre-dado de la disposición afectiva, para la acción volitiva en sentido estricto, la decisión; en esa medida, forman parte de la disposición para actuar.

Las consideraciones aquí propuestas permiten ver un mapa de ruta para la elucidación de una forma concreta e integral de la idea de "autoconocimiento" desde una perspectiva fenomenológica. Asimismo sugerimos la plausibilidad del análisis a partir de un ejercicio descriptivo concreto de la meditación sobre la formación del carácter, como apertura de sentido de la subjetividad concreta en su situación vital y su "decisión”.

\section{Bibliografía}

Brough, John B., 2005, "The Emergency of an absolute conscioussness in Husserl's early writtings on time-consciousness", en Rudolf Bernet, Donn Welton and Gina Zavota, Edmund Husserl, Critical Assessments of Leading Philosophers, vol. III, Nueva York, Routledge, pp. 247-272. 
Dodd, James, 2010, "Death and Time in Husserl's C-Manuscripts", en Dieter Lohmar y Ichiro Yamaguchi, On Time. New Contributions to the Husserlian Phenomenology of Time, Springer, Phänomenologica, 197, pp. 51-70.

Gross, Alexis, 2011, "Wilhelm Dilthey y la fundamentación psicologista de las ciencias humanas, en C. B. González y P. Perkins (comps.), Estudios de Fenomenología y Hermenéutica V. Vida $y$ concepto. Conmemorando el centenario de Wilhelm Dilthey (1833-1911), Paraná, Círculo de Fenomenología y Hermenéutica de Santa Fe-Paraná, pp. 7-14.

Husserliana I, 1973, Cartesianische Meditationen und Pariser Vorträge, S. Strasser, (ed.), La Haya, Martinus Nijhoff.

Husserliana III/1, 1977, Ideen zu einer reinen Phänomenologie und phänomenologischen Philosophie. Erstes Buch: Allgemeine Einführungin die reine Phänomenologie 1. Halbband: Text der 1.-3. Auflage — Nachdruck, Karl Schuhmann (ed.), La Haya, Martinus Nijhoff.

Husserliana IV, 1984, Ideen zu einer reinen Phänomenologie und phänomenologischen Philosophie, Zweites Buch: Phänomenologische Untersuchungen zur Konstitution, Marly Biemel (ed.), Kluwer Academic Publishers B.V.

Husserliana Materialien, Band VIII. 2006, Späte Texte über Zeitkonstitution (1929-1934). Die C-Manuskripte, Dieter Lohmar (ed.), Nueva York, Springer.

Manuscritos inéditos estudiados y citados de acuerdo a la ordenación del Archivo Husserl de la Universidad Católica de Lovaina.: Ms. A VI 8 I - II, Ms. A VI 12 I-II, Ms. A VI 34, Ms. E III 4, Ms. E III 6, Ms. M III 3, I-III.

Mezzanzanica, Massimo, 2011, "Philosophie der Erfahrung und Erneuerung des Apriori: Dilthey und Helmholtz", en Hans-Ulrich, Rudolf A. Makkreel y Riccardo Pozzo (eds.), Recent Cont- 
ributions to Dilthey Philosophy of the Human Sciences, Stuttgart, Frooman Holzboog Verlag, pp. 59-81.

Schumman, Karl, 1997, Husserl-Cronik, Denk-und Lebensweg Edmund Husserls, Husserliana Dokumente 1, La Haya, Martinus Nijoff.

Taylor, Charles, 2006, Las fuentes del yo, Ana Lizón (trad.), Barcelona, Paidós.

Walton Roberto, 2004, "Horizonticidad y Juicio", Anuario Filosófico XXXVII/I, núm. 78, pp. 197-242.

Zirión, Antonio, 2009, "El resplandor de la afectividad", en Acta Fenomenológica Latinoamericana, v. III, Morelia, Círculo Latinoamericano de Fenomenología, PUCP-UMSNH.

(Artículo recibido el 7 de enero de 2014; aceptado el 25 de febrero de 2014) 